\title{
Human Bone Marrow Mesenchymal Stem Cells Promote Gastric Cancer Growth via Regulating $c-M y c$
}

\author{
Bin Chen, ${ }^{1}$ Jing Yu, ${ }^{1,2}$ Qianqian Wang, ${ }^{1}$ Yuanyuan Zhao, ${ }^{1}$ Li Sun, ${ }^{1}$ Changgen Xu, ${ }^{3}$ \\ Xiangdong Zhao, ${ }^{3}$ Bo Shen, ${ }^{4}$ Mei Wang, ${ }^{1}$ Wenrong $X u\left(D,{ }^{1}\right.$ and Wei Zhu ${ }^{1}{ }^{1}$ \\ ${ }^{1}$ School of Medicine, Jiangsu University, Zhenjiang, Jiangsu, China \\ ${ }^{2}$ The Xuyi People's Hospital, Xuyi, Jiangsu, China \\ ${ }^{3}$ Zhenjiang Provincial Blood Center, Zhenjiang, Jiangsu, China \\ ${ }^{4}$ Department of Oncology, Jiangsu Cancer Hospital Affiliated to Nanjing Medical University, Nanjing, Jiangsu, China
}

Correspondence should be addressed to Wei Zhu; zhuwei@ujs.edu.cn

Bin Chen and Jing Yu contributed equally to this work.

Received 27 January 2018; Revised 9 April 2018; Accepted 2 May 2018; Published 18 July 2018

Academic Editor: Hua Tan

Copyright (C) 2018 Bin Chen et al. This is an open access article distributed under the Creative Commons Attribution License, which permits unrestricted use, distribution, and reproduction in any medium, provided the original work is properly cited.

The clinical application of human bone marrow mesenchymal stem cells (hBM-MSCs) has generated a great deal of interest because of their potential use in regenerative medicine and tissue engineering. However, safety concerns over hBM-MSCs limit their clinical application. In this study, we observed that hBM-MSC-conditioned medium (hBM-MSC-CM) promotes gastric cancer development via upregulation of c-Myc. Our results showed that c-Myc was upregulated in MGC-803 and BGC-823 cells after hBM-MSC-CM treatment. Moreover, we found that the c-Myc inhibitor JQ1 and c-Myc siRNA decreased the expression of c-Myc in hBM-MSC-CM-treated tumor cells in vitro. Additionally, hBM-MSC-CM enhanced the migration and glucose uptake of gastric cancer cells. In vivo studies showed that JQ1 inhibited hBM-MSC-CM-induced gastric cancer growth. These results indicated that hBM-MSC-CM induced gastric cancer growth via upregulation of c-Myc, which may be a potential risk factor and/or a therapeutic target for clinical applications.

\section{Introduction}

Due to their multidirectional differentiation capacity, mesenchymal stem cells (MSCs) have been widely used in tissue engineering and cell replacement therapy [1]. However, studies have increasingly demonstrated that human bone marrow MSCs (hBM-MSCs) can promote tumor growth and metastasis $[2,3]$, making safety of hBM-MSCs in clinical applications controversial.

Because of their promise for clinical applications, a variety of models have been used to prove the safety and effectiveness of hBM-MSCs $[4,5]$. Some previous clinical studies have concluded that hBM-MSCs do not pose an obvious risk of tumorigenesis when used to treat cartilage injuries or other diseases [6, 7]. However, other studies have found that hBM-MSCs promote tumor proliferation, migration, and stemness in vitro and that hBM-MSCs promote tumor development in vivo $[3,8,9]$. Therefore, there is an urgent need to identify the factors from hBM-MSCs that promote tumor growth.

In this study, we found that hBM-MSC-CM caused gastric cancer cells to upregulate c-Myc expression, which is a well-known oncogene that is involved in tumor initiation and development. Abnormal c-Myc activation is responsible for a range of human cancers, including neuroblastoma [10], lung carcinoma [11], and gastric carcinoma [12]. By promoting c-Myc expression, hBM-MSC-CM increased the metabolism, migration, and proliferation of gastric cancer cells. Furthermore, we showed that the c-Myc inhibitor JQ1 inhibits the tumor-promoting effects of hBM-MSC-CM. Thus, we show that hBM-MSC-CM can upregulate c-Myc expression in gastric cancer cells, which may be a key factor in carcinogenesis and, therefore, a potential target for cancer prevention. 


\section{Materials and Methods}

2.1. Xenograft Tumor Model. This study and its consent procedure were approved by the local ethics committee of Jiangsu University (Jiangsu, China). BALB/c-nu/nu male mice ( $n=30$; aged 4-5 weeks) were purchased from SLAC Laboratory Animal (Shanghai, China) and maintained in pathogen-free conditions with sterilized chow and autoclaved water. The animals were randomly divided into five groups ( $n=6$ mice per group). MGC- 803 cells were treated with either Dulbecco's modified Eagle's medium (DMEM; Gibco/Life Technologies, Carlsbad, CA, USA), dimethyl sulfoxide (DMSO; Sigma-Aldrich, St. Louis, MO, USA), hBMMSC-conditioned media (hBM-MSC-CM), JQ1 $(0.8 \mu \mathrm{M})$, or hBM-MSC-CM + JQ1 for $48 \mathrm{~h}$. In the hBM-MSC-CM+ JQ1 group, MGC-803 cells were pretreated with JQ1 for 4-6h, washed three times with phosphate-buffered saline (PBS), and then treated with hBM-MSC-CM+JQ1. Then, subcutaneous xenografts were established by injection of $2 \times 10^{6}$ MGC-803 cells in $200 \mu \mathrm{L}$ of PBS per mouse. Once tumors formed, tumor sizes were measured using Vernier calipers and tumor volumes were calculated using the following formula: tumor volume $=$ length $\times$ width $^{2} / 2$. The experiment was stopped on day 30 , when all mice were euthanized.

2.2. Cell Culture. Healthy donor-derived bone marrow cells were collected at the Affiliated Hospital of Jiangsu University, and all protocols were approved by the local ethics committee of the Affiliated Hospital of Jiangsu University, China. Additionally, informed consent was received from all donors. The bone marrow cells were diluted in an equal volume of PBS, isolated with $1.077 \mathrm{~g} / \mathrm{mL}$ Ficoll, and centrifuged at $800 \times \mathrm{g}$ for $20 \mathrm{~min}$. The cells were then rinsed with PBS and cultured in DMEM containing 10\% fetal bovine serum (FBS; Gibco) at $37^{\circ} \mathrm{C}$ in a humidified incubator infused with $5 \% \mathrm{CO}_{2}$; adherent cells were collected after 5 days. The human gastric cancer cell lines MGC-803 and BGC-823 and normal line GES-1 were purchased from the Cell Bank of Type Culture Collection of Chinese Academy of Sciences (Beijing, China) and were maintained in DMEM supplemented with $10 \%$ $\mathrm{FBS}$ at $37^{\circ} \mathrm{C}$ in $5 \% \mathrm{CO}_{2}$.

\subsection{Preparing hBM-MSC-CM and Coculture with Gastric} Cancer Cells. When hBM-MSCs had grown to $70 \%$ confluency, they were washed with PBS and incubated with fresh medium for $48 \mathrm{~h}$. Then, the supernatant was collected, filtered through a $0.22 \mu \mathrm{m}$ filter, and diluted at $1: 1$ with DMEM supplemented with 10\% FBS (hBM-MSC-CM). MGC-803 and BGC-823 cells were washed with PBS and then treated with DMEM, DMSO, hBM-MSC-CM, JQ1 $(0.8 \mu \mathrm{M})$, or $\mathrm{hBM}-\mathrm{MSC}-\mathrm{CM}+\mathrm{JQ} 1$ at $37^{\circ} \mathrm{C}$ in $5 \% \mathrm{CO}_{2}$ for $48 \mathrm{~h}$ prior to being collected for use in subsequent experiments.

2.4. Transwell Migration Assay. Transwell migration assays were performed to evaluate the effect of JQ1 or c-Myc knockdown on the hBM-MSC-CM-induced migration of gastric cancer cells. In the experiment using JQ1, $1 \times 10^{5}$ MGC-803 or BGC-823 cells were plated in six-well plates (Corning Inc., Corning, NY, USA) and then treated with
DMEM, DMSO, hBM-MSC-CM, JQ1 (0.8 $\mu \mathrm{M})$, and hBMMSC-CM + JQ1. In the experiment using c-Myc knockdown, the cells after c-Myc knockdown were cocultured with hBMMSC-CM. After $48 \mathrm{~h}$, the cells were collected by centrifugation $(800 \mathrm{rpm} / \mathrm{min})$ and $8 \times 10^{4}$ cells were seeded in the upper wells of a Transwell chamber in $200 \mu \mathrm{L}$ of serumfree DMEM. The lower compartment of the chamber was filled with $500 \mu \mathrm{L}$ DMEM supplemented with 10\% FBS. The cells were incubated for $8 \mathrm{~h}$, and after incubation, we used a cotton swab to remove the cells that did not migrate. Migrated cells were fixed with $4 \%$ formaldehyde for $30 \mathrm{~min}$, stained with crystal violet, and then photographed. For quantitation, three random fields from each well were counted under a microscope (Ti-S; Nikon, Tokyo, Japan), and each experiment was independently repeated in triplicate.

2.5. Glucose Uptake Assay. Glucose uptake assays were performed to evaluate the effect of hBM-MSC-CM on the glucose utilization of gastric cancer cells. Briefly, $1 \times 10^{5}$ MGC-803 or BGC-823 cells were plated in six-well plates (Corning Inc.) and then treated with DMEM, DMSO, hBM-MSC-CM, JQ1 $(0.8 \mu \mathrm{M})$, and hBM-MSC-CM+JQ1. After $48 \mathrm{~h}$, the cells were collected by centrifugation $(800 \mathrm{rpm} / \mathrm{min})$ and $1 \times 10^{6}$ cells $/ \mathrm{mL}$ were seeded in 48 -well plates and incubated for $8 \mathrm{~h}$ in DMEM. Then, a clinical chemistry analyzer (Xunda, XD811, Shanghai, China) and the hexokinase method were used to detect supernatant glucose concentrations.

2.6. Cell Viability Assay. We performed 3-[4,5-dimethylthiazol-2yl] diphenyltetrazolium bromide (MTT) assays to assess the half maximal inhibitory concentration (IC50) of JQ1. Cells were seeded into 96-well plates (Corning Inc.) at a density of $2 \times 10^{3}$ per well and incubated overnight at $37^{\circ} \mathrm{C}$ in $5 \% \mathrm{CO}_{2}$. Then, the plates were incubated with DMSO or different JQ1 concentrations $(0.4$ and $0.8 \mu \mathrm{M})$ and cultured for 24,48 , or $72 \mathrm{~h}$. MTT $(5 \mathrm{mg} / \mathrm{mL})$ was added to the cells for $4 \mathrm{~h}$ at $37^{\circ} \mathrm{C}$. After $4 \mathrm{~h}$, we added DMSO to terminate the reaction; results were determined by measuring optical density at $490 \mathrm{~nm}$ with a multiwell plate reader (FLx800, BioTek, Winooski, VT, USA). Each experiment was repeated three times.

2.7. Western Blot Analysis. Total proteins were extracted from cells using RIPA lysis buffer (Invitrogen, Carlsbad, CA, USA). After determining protein concentration, $20 \mu \mathrm{g}$ of total protein from each sample was separated with $12 \%$ sodium dodecyl sulfate polyacrylamide gel electrophoresis and then transferred to polyvinylidene fluoride membranes (Millipore, Billerica, MA, USA). The membranes were blocked with 5\% nonfat milk and incubated with antihuman c-Myc (dilution, 1:300; 10057-1-AP, Proteintech, Chicago, IL, USA), Bcl-2 (dilution, $1: 200 ; 2870$, Cell Signaling Technology, USA), Bax (dilution, 1:200; BS2538, Bioworld, USA), cyclin-D1 (dilution, 1:500; BS1741, Bioworld, USA), and Glut1 (dilution, 1:1000; FO6231163, Wanleibio, Shenyang, China) antibodies overnight at $4^{\circ} \mathrm{C}$ and then incubated with goat anti-mouse (dilution, 
1:2000; CW0102, CWBIO, Beijing, China) and goat antirabbit (dilution, 1:2000; CW0103, CWBIO, Beijing, China) secondary antibodies for 1 hour at $37^{\circ} \mathrm{C}$. The blots were visualized using the enhanced chemiluminescent detection system (Amersham, Amersham, UK) and analyzed using Image-Pro Plus version 5.1 (Media Cybernetics Inc., Rockville, MD, USA).

2.8. Cell Cycle Analysis. The effects of hBM-MSC-CM and JQ1 on cell cycle distributions were measured using a flow cytometer (FACSCalibur, BD Biosciences, Franklin Lakes, NJ, USA). Briefly, $1 \times 10^{5}$ cells per well were seeded into six-well plates, incubated overnight, and then treated with DMEM, DMSO, hBM-MSC-CM, JQ1 $(0.8 \mu \mathrm{M})$, and hBMMSC-CM+JQ1. After $48 \mathrm{~h}, 1 \times 10^{6}$ cells per well were collected by centrifugation and stained with propidium iodide in PBS for $30 \mathrm{~min}$ at $4^{\circ} \mathrm{C}$ in the dark before being analyzed with flow cytometry.

2.9. Colony Formation Assay. MGC- 803 or BGC- 823 cells were treated with DMEM, DMSO, hBM-MSC-CM, JQ1, and hBM-MSC-CM+JQ1 for $48 \mathrm{~h}$, and then cells were seeded at 500 cells per well in $6 \mathrm{~cm}$ well plates in triplicate. After 7 days of growth, the cells were washed with PBS, fixed with $4 \%$ paraformaldehyde, and stained with crystal violet. We counted the number of colonies per well under a dissecting microscope.

2.10. c-Myc Knockdown. c-Myc siRNA (50 nM) (5'-GGACTA TCCTGCTGCCAAG- $\left.3^{\prime}\right)$ and negative control (NC) $(50 \mathrm{nM})$ were purchased from Guangzhou RiboBio. siRNA were transfected into MGC-803 with Lipofectamine ${ }^{\circledR} 2000$ reagent (Invitrogen; Thermo Fisher Scientific Inc.) according to the instructions.

2.11. Statistical Analysis. All data analyses were performed using GraphPad Prism 6 (Graph Software, La Jolla, CA, USA). Differences between groups were analyzed using oneway analysis of variance. The Kruskal-Wallis $H$ test was used to analyze differences between in vivo tumor growths. A $P$ value $<0.05$ was considered statistically significant.

\section{Results}

3.1. HBM-MSC-CM Increased c-Myc Expression in Gastric Cancer Cells. The c-Myc oncogene has been reported to play important roles in gastric cancer; thus, we examined c-Myc levels in the gastric cancer cell lines MGC-803 and BGC-823 and normal line GES- 1 with Western blot. GES- 1 cells showed the lowest c-Myc expression, and the BGC-823 cells showed the highest c-Myc expression (Figure 1(a)). Next, we investigated whether treating the gastric cancer cells with hBMMSC-CM for $48 \mathrm{~h}$ affected c-Myc protein levels. Compared with the untreated groups, c-Myc levels were increased in both MGC-803 and BGC-823 cells after hBM-MSC-CM treatment (Figure 1(b)). The upregulation of c-Myc expression in MGC-803 cells could maintain 12 hours after withdrawal of hBM-MSC-CM (Figure 1(c)). JQ1 has been shown to have antiproliferative effects in many cancers, primarily through inhibition of c-Myc [13]. MTT assays showed that $0.8 \mu \mathrm{M}$ JQ1 for $72 \mathrm{~h}$ has significantly antiproliferative effects on MGC-803 (Figure 1(d)). Then, we used JQ1 at $0.8 \mu \mathrm{M}$ concentration in the following experiment. Western blot analysis indicated that hBM-MSC-CM treatment can significantly increase the expression level of c-Myc protein, and the effect of hBM-MSC-CM upregulation of c-Myc in MGC-803 and BGC-823 cells can also be suppressed by JQ1 (Figures 1(e) and 1(f)).

\subsection{JQ1 Inhibited the Gastric Cancer Cell Proliferation In} Vitro. The effect of hBM-MSC-CM and hBM-MSC-CM + JQ1 on the proliferation of gastric cancer cells was analyzed with colony formation and MTT assays. The colony formation assays showed that the hBM-MSC-CM+JQ1 group formed fewer colonies than the hBM-MSC-CM group $(P<0.001$, Figures $2(\mathrm{a})-2(\mathrm{~d}))$. Consistent with the colony formation assays, MTT assays showed that JQ1 inhibited the proliferation rate of MGC-803 and BGC-823 cells $(P<0.001$, Figures 2(e) and 2(f)).

3.3. Effect of hBM-MSC-CM on Cell Cycle Progression and Apoptosis. Cell cycle analysis revealed that JQ1 treatment increased the percentage of G1-phase cells from $51.42 \%$ to $61.49 \%$, while the fraction of S-phase cells decreased from $30.31 \%$ to $22.3 \%$, which were not statistically significant changes (Figure 3(a)). Additionally, hBM-MSC-CM had no effect on the cyclin-D1 expression or the apoptosis rates of MGC-803 cells (Figures 3(b) and 3(c)); however, JQ1 inhibited cyclin-D1 expression.

3.4. HBM-MSC-CM Promoted Migration and Glucose Uptake in MGC-803 and BGC-823 Cells. To determine whether in vitro hBM-MSC-CM treatment promoted migration and glucose uptake in MGC-803 and BGC-823 cells (common features of cells with c-Myc upregulation), we performed Transwell migration, Western blot, and glucose uptake assays. Transwell migration assays showed that hBM-MSCCM increased the migratory ability of MGC-803 and BGC823 cells and that JQ1 significantly inhibited these effects (both $P<0.001$; Figures 4(a) $-4(\mathrm{~d})$ ). In order to further verify the hBM-MSC-CM promoting gastric cancer development via upregulation of c-Myc directly, we chose to use c-Myc siRNA which can inhibit c-Myc expression directly. c-Myc siRNA can inhibit the expression of c-Myc in MGC-803 cells (Figure 4(e)). Reducing the expression of c-Myc in MGC-803 cells can inhibit the effect of hBM-MSC-CM on tumor migration (Figures 4(f) and 4(g)). Western blot and glucose uptake assays also showed that JQ1 inhibited hBM-MSCCM-induced increases in Glut1 expression in MGC-803 and glucose uptake in MGC-803 and BGC-823 cells (Figures 4(h)-4(j)).

\subsection{HBM-MSC-CM Increased Gastric Cancer Growth In} Vivo. To determine whether hBM-MSC-CM promoted in vivo tumor growth, we injected MGC-803 cells treated with DMEM, DMSO, JQ1, hBM-MSC-CM, and hBMMSC-CM+JQ1 for $48 \mathrm{~h}$ into BALB/c-nu/nu mice. Consistent with our previous studies, hBM-MSC-CM promoted tumor growth in vivo. Additionally, tumor volumes and weights of the JQ1 and hBM-MSC-CM+JQ1 groups were 


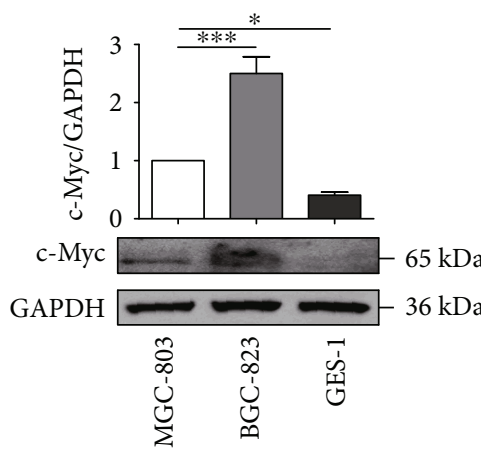

(a)
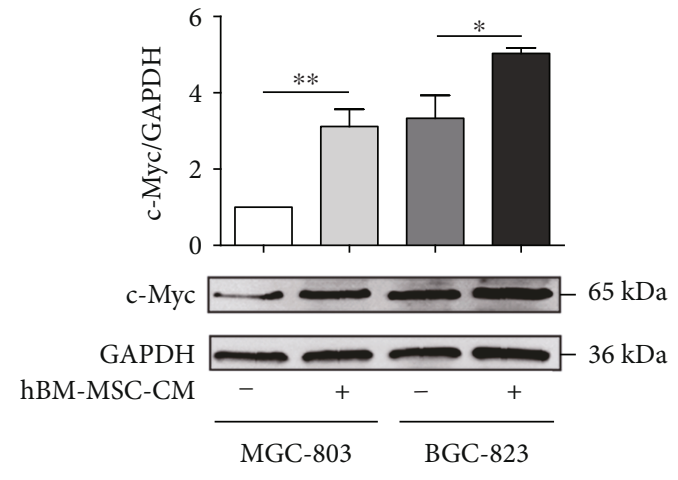

(b)

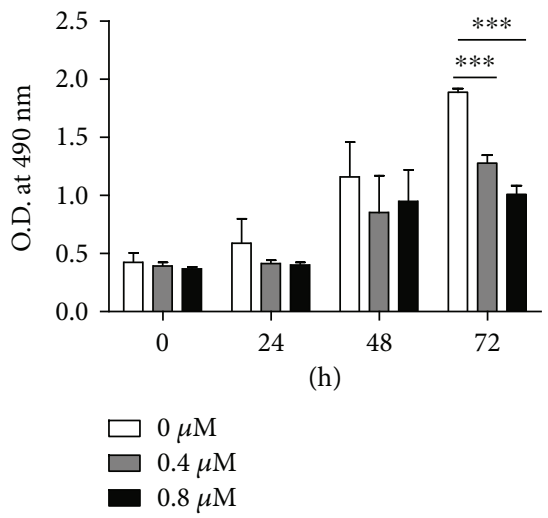

(d)

MGC-803
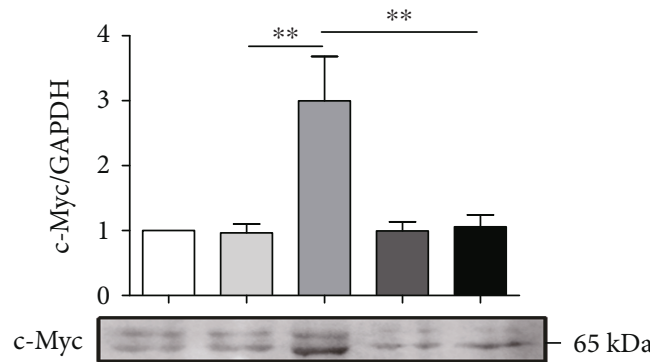

GAPDH

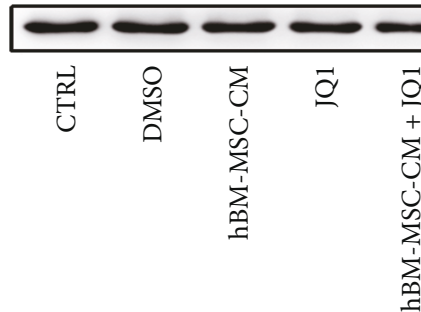

(e)

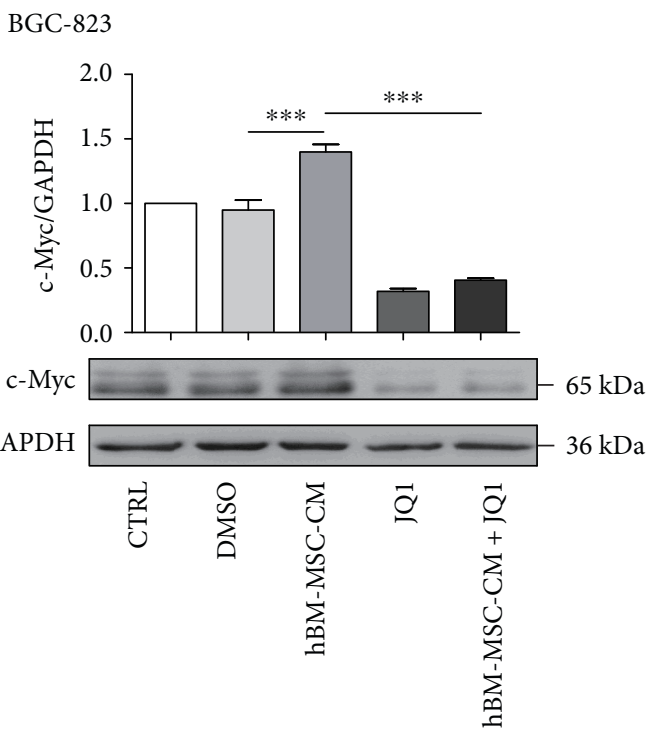

(f)

FIGURE 1: hBM-MSC-CM increased c-Myc expression in gastric cancer cells. (a) c-Myc protein levels in MGC-803, BGC-823, and GES-1 were detected by Western blot analysis. ${ }^{*} P<0.05,{ }^{* * *} P<0.001$. (b) c-Myc levels after $48 \mathrm{~h} \mathrm{hBM-MSC-CM}$ treatment. ${ }^{*} P<0.05,{ }^{* *} P<0.01$ (c) cMyc protein levels maintained after withdrawal of hBM-MSC-CM treatment were detected by Western blot analysis. (d) MGC-803 cells were treated with 0.4 and $0.8 \mu \mathrm{M}$ JQ1 for 24, 48, and $72 \mathrm{~h} .{ }^{* * *} P<0.001$. (e, f) Compared with hBM-MSC-CM, hBM-MSC-CM + JQ1 decreased cMyc expression in MGC-803 or BGC-823 cells. ${ }^{* *} P<0.01,{ }^{* * *} P<0.001$.

smaller than those of the hBM-MSC-CM and control groups $(P<0.001$; Figures 5(a) $-5(\mathrm{c}))$. Western blotting of xenograft tumor tissue showed that hBM-MSC-CM could not maintain the upregulation of c-Myc in vivo (Figure 5(d)).

\section{Discussion}

Previous studies have suggested that MSCs are closely associated with tumor progression and growth [3]; thus, there are 


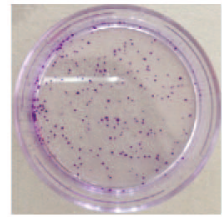

CTRL

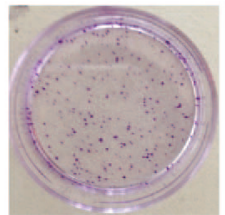

DMSO

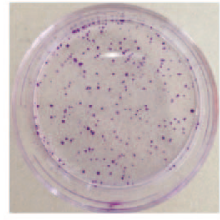

hBM-MSC-CM

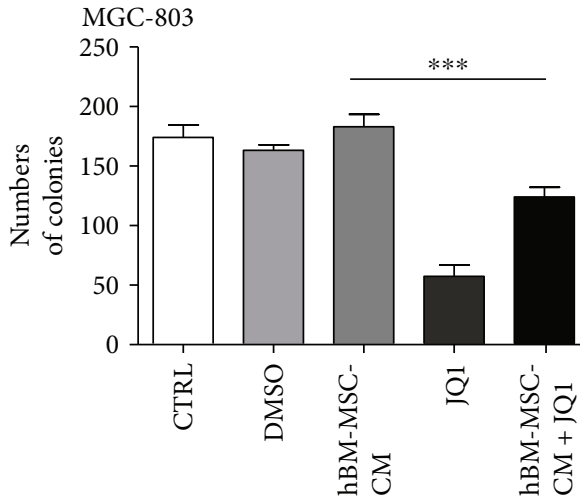

(a)

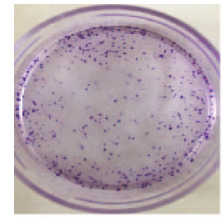

CTRL

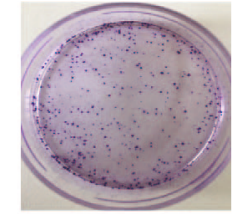

DMSO

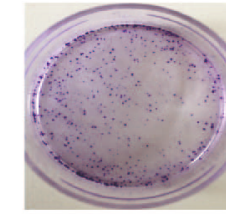

hBM-MSC-CM

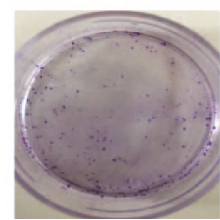

JQ1

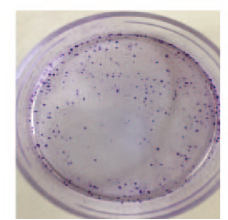

hBM-MSC-CM + JQ1

(c)

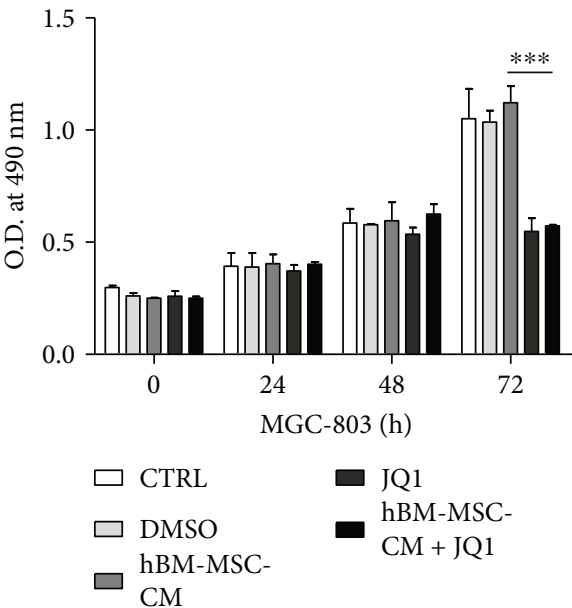

(e)

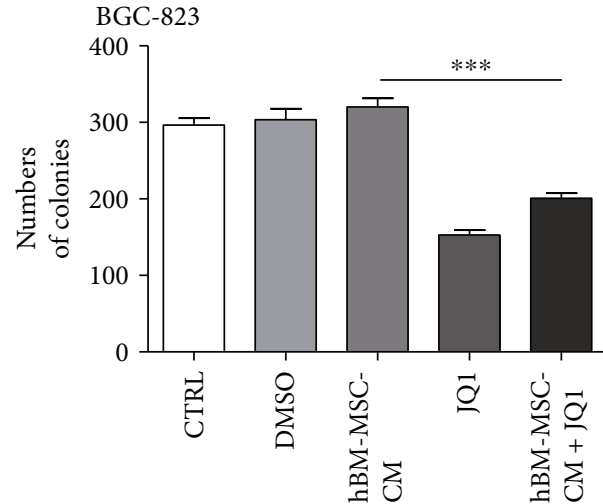

(d)

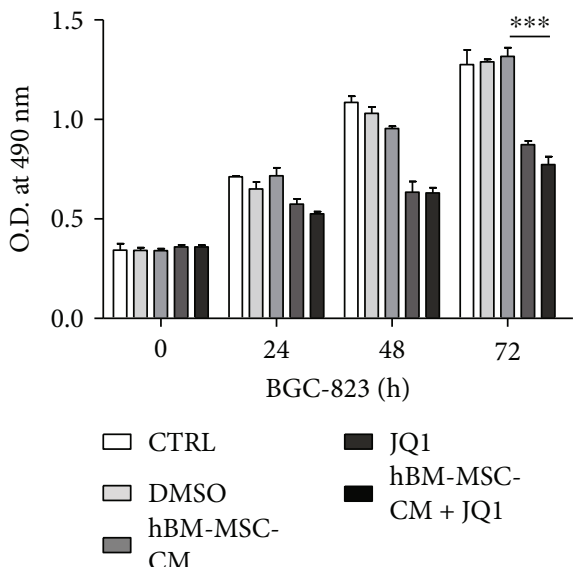

(f)

FIGURE 2: JQ1 inhibited the gastric cancer cell proliferation in vitro. (a, c) JQ1 reduced MGC-803 or BGC-823 colony formation rates with and without hBM-MSC-CM. (b, d) The colony formation rate of the hBM-MSC-CM + JQ1 group was lower than that of the hBM-MSC-CM group. ${ }^{* * *} P<0.001$ by a one-way analysis of variance. (e, f) MTT assays showed that JQ1 decreased the proliferation rate of MGC-803 or BGC-823 cells compared with the hBM-MSC-CM group. ${ }^{* * *} P<0.001$ by a one-way analysis of variance. 


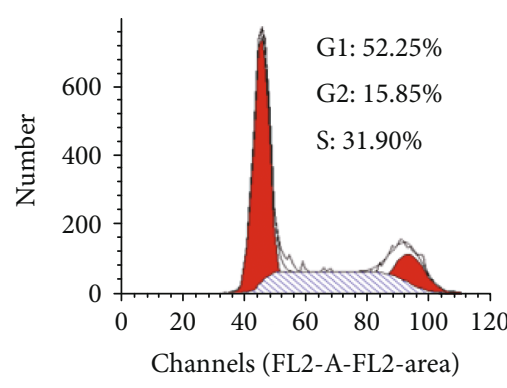

CTRL

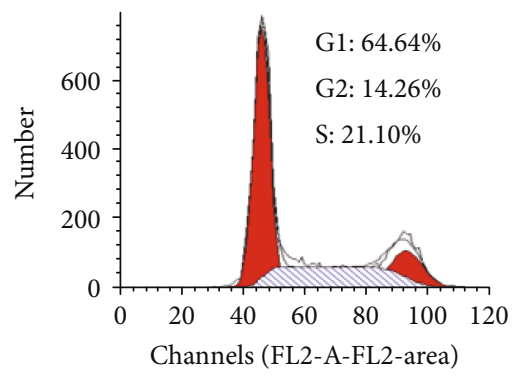

JQ1

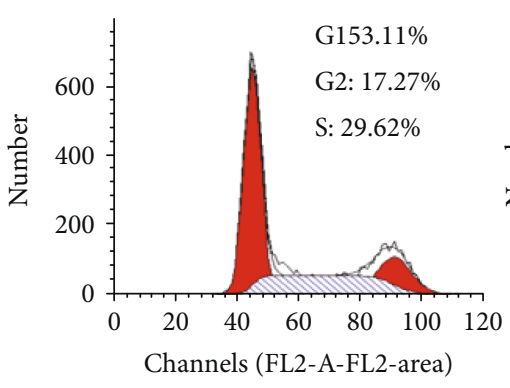

DMSO
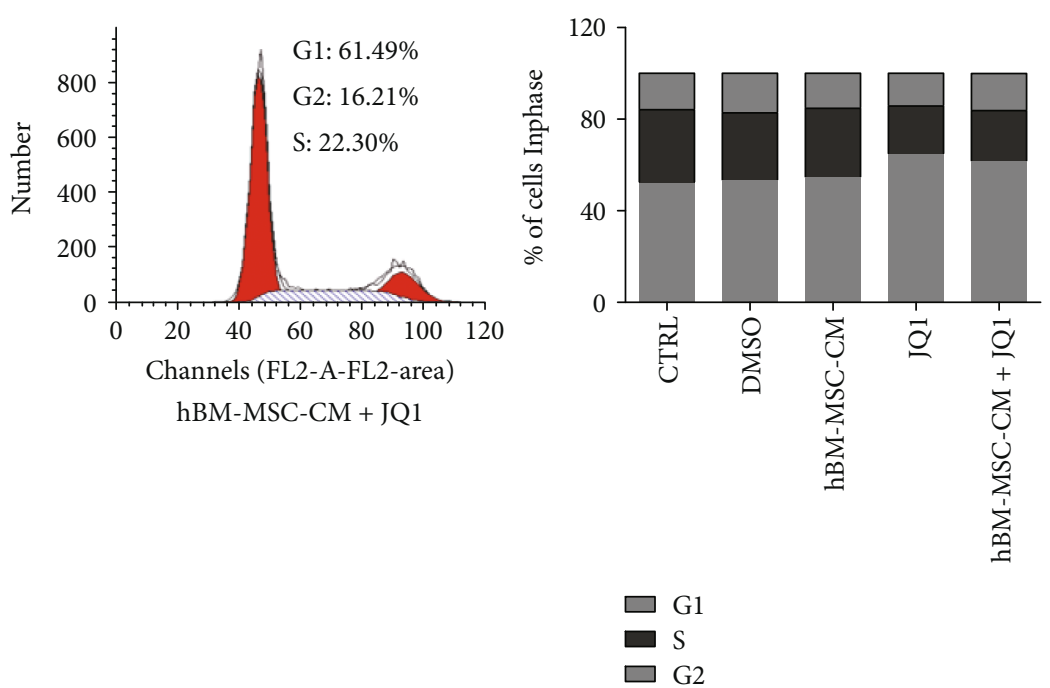

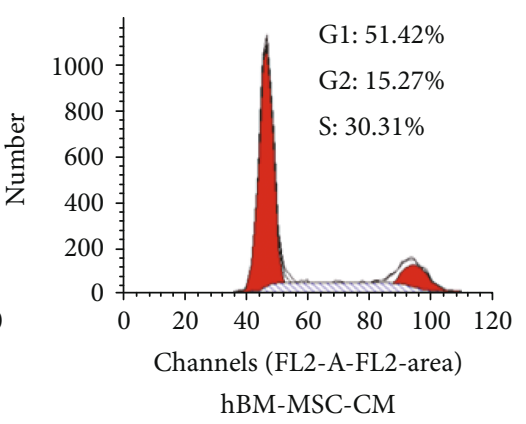

- S

(a)

\section{MGC-803}

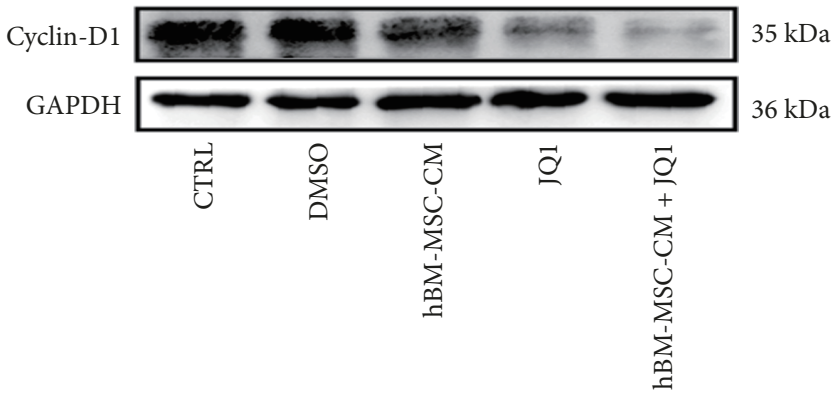

(b)

MGC-803

\section{MGC-803}

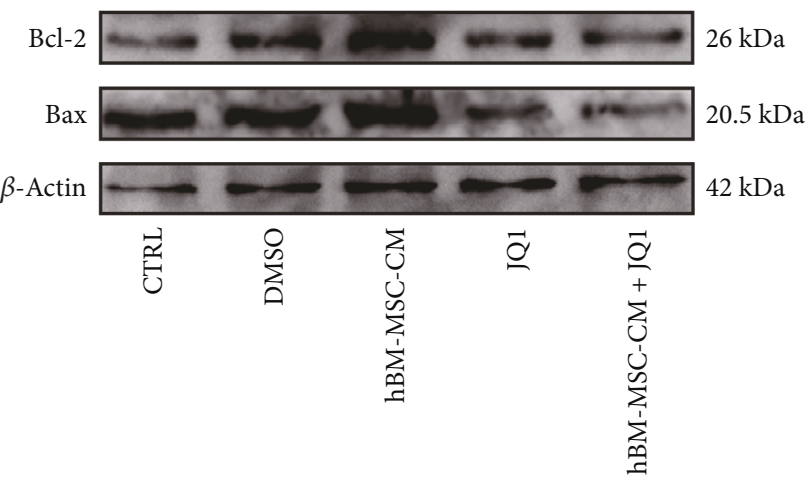

(c)

Figure 3: Effect of hBM-MSC-CM on cell cycle progression and apoptosis in gastric cancer cells. (a) hBM-MSC-CM + JQ1 induced a G1 arrest compared with the hBM-MSC-CM group; the changes in cell cycle distribution were not significant after three experiments. (b) Western blot analysis of cyclin-D1 expression in hBM-MSC-CM + JQ1-treated MGC-803 cells. (c) Western blot analysis of Bcl-2 and Bax expression in hBM-MSC-CM + JQ1-treated MGC-803 cells.

legitimate safety concerns that limit their clinical application. Our previous study revealed that MSCs promote tumor growth in vivo, which suggested that these cells secrete mitogenic paracrine factors $[8,9]$. Jung et al. demonstrated that the MSCs recruited by tumors facilitate metastasis [14], and $\mathrm{Li}$ et al. revealed that gastric cancer-derived MSCs enhanced the proliferation and migration of gastric cancer cell lines [15]. Djouad et al. reported that in vivo tumor growth was increased when melanoma cells were coinjected with MSCs [16]. Interestingly, Karnoub et al. found that in situ breast cancer was unchanged in the absence of MSCs; however, the migratory ability of tumor cells was significantly increased when exogenous MSCs were added [17]. In this study, we chose MGC-803 and BGC-823 cells as representative cell lines to further study the mechanism through which hBM-MSC-CM promotes tumor growth. 


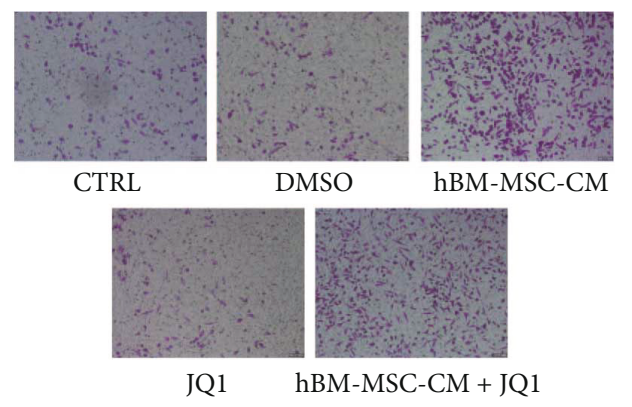

(a)

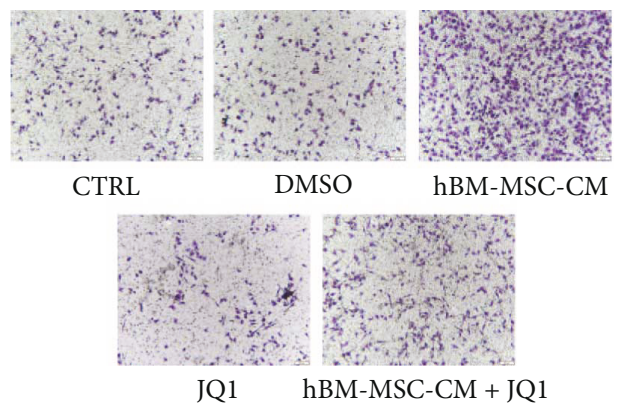

(c)

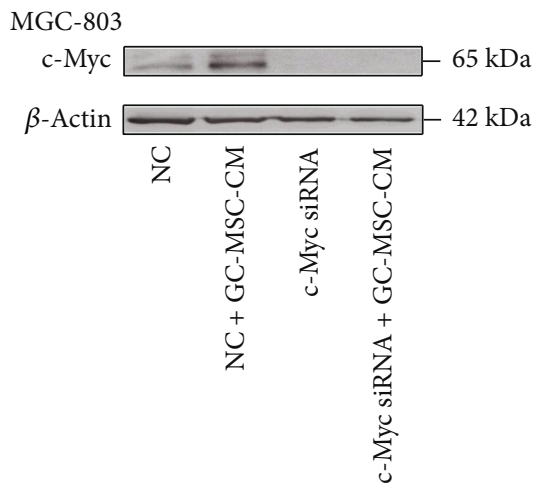

(e)

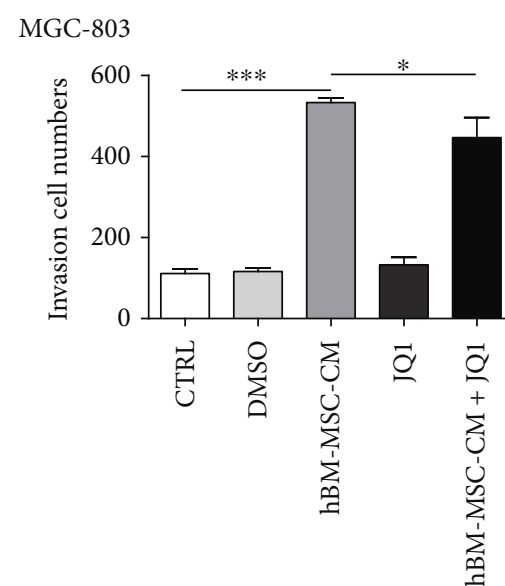

(b)

BGC- 823

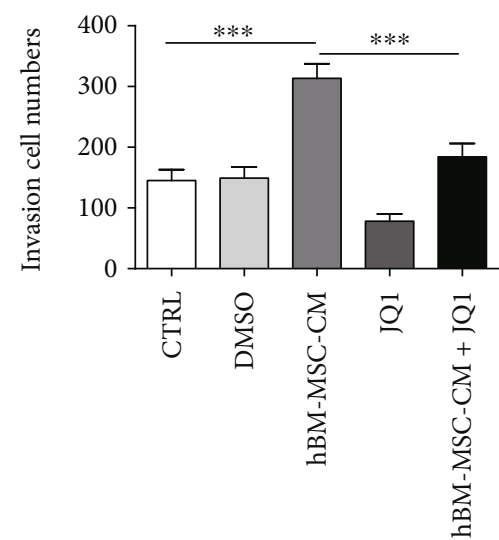

(d)
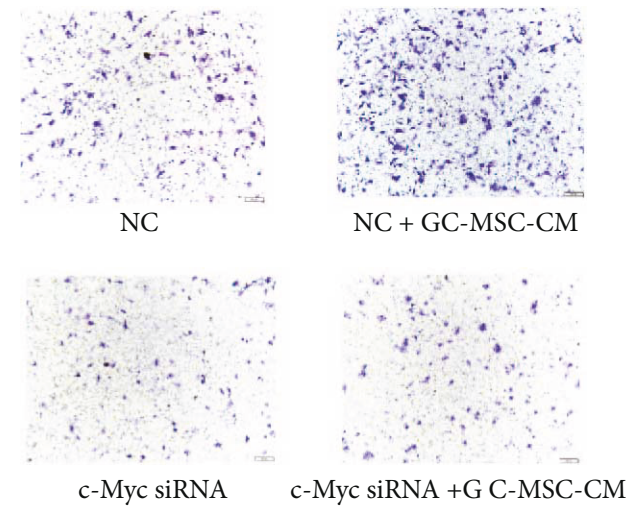

(f)

Figure 4: Continued. 


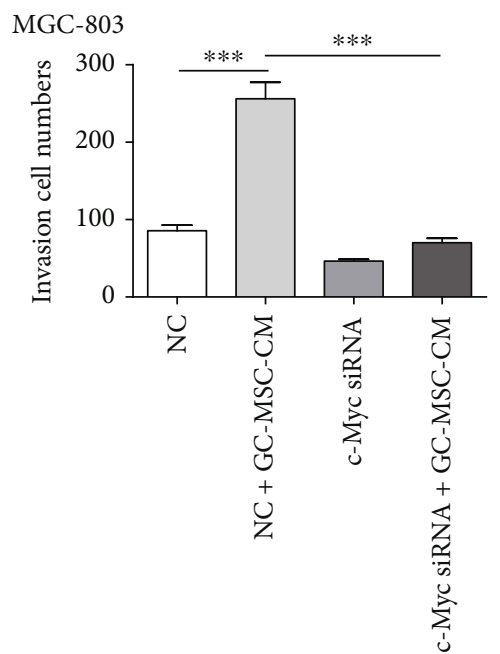

(g)

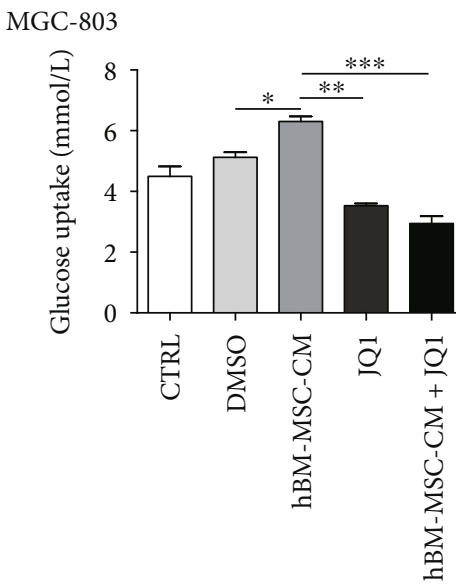

(i)

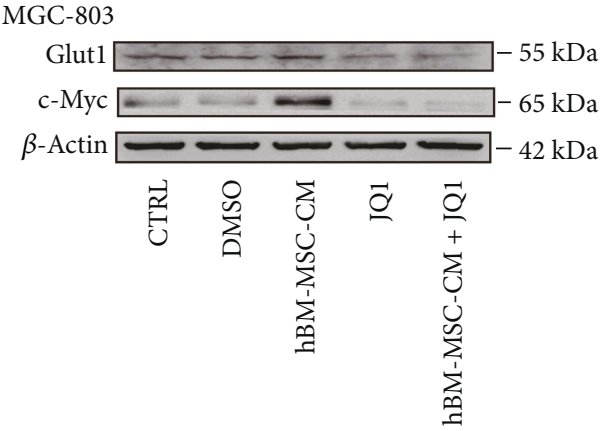

(h)

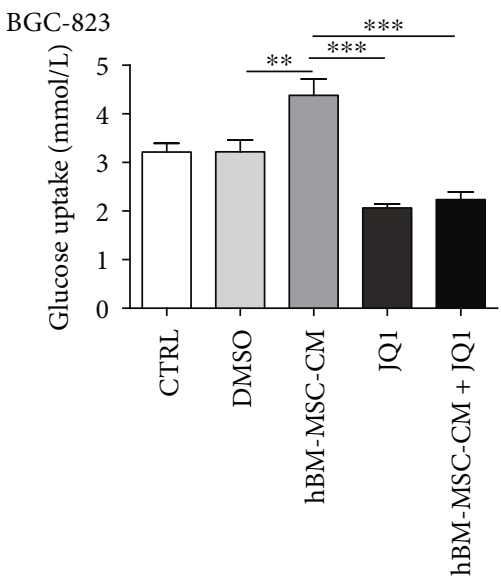

(j)

FIGURE 4: hBM-MSC-CM promoted migration and glucose uptake of MGC-803 and BGC-823 cells. (a, c) Transwell migration assays showed that hBM-MSC-CM increased the migratory ability of gastric cancer cells and that this activity was inhibited by JQ1 (scale bar: $100 \mu \mathrm{m}$ ). (b, d) The numbers of migrated cells were analyzed by a one-way analysis of variance $\left({ }^{*} P<0.05,{ }^{* * *} P<0.001\right)$. (e) c-Myc siRNA can inhibit the expression of c-Myc induced by hBM-MSC-CM in MGC-803 cells. (f, g) The effect of hBM-MSC-CM on MGC-803 cell migration can be suppressed by c-Myc siRNA ( $\left.{ }^{* *} P<0.001\right)$. (h) hBM-MSC-CM-induced Glut1 upregulation in MGC-803 cells was detected with Western blot. (i, j) The glucose uptake of MGC-803 and BGC-823 cells after the indicated treatments was detected using a clinical chemistry analyzer $\left({ }^{*} P<0.05,{ }^{* *} P<0.01,{ }^{* * *} P<0.001\right)$.

We explored the potential risks of using hBM-MSCs in clinical applications. Although the tumor-promoting activity of patient-derived hBM-MSCs may be more dramatic, patient-derived hBM-MSCs could not be used in clinical applications; thus, we used healthy donor-derived hBMMSCs. Our preliminary studies showed that the mitogenic activity of hBM-MSCs on tumor cells was primarily through paracrine signaling [8]. So, we subsequently focused on the potential factors from hBM-MSCs that promoted cancer progression, as these comprised the molecular signature of potential targets that could allow their clinical application.

c-Myc is a protooncogene that is mutated in approximately $20 \%$ of human cancers and has particularly important functions in gastric carcinogenesis [18]. In the present study, we found that c-Myc expression was increased in hBM-MSC-CM-treated gastric cancer cells, suggesting that
hBM-MSC-CM may promote tumor growth by upregulating c-Myc. Moreover, c-Myc has been shown to maintain normal adult stem cells and tumor stem cells [19]. It is worth noting that the effects of hBM-MSC-CM on tumor cell proliferation and cell cycle progression were not obvious in vitro. The experimental results showed that the upregulation of c-Myc expression in tumor cells could maintain 12 hours after withdrawal of hBM-MSC-CM. However, the inhibitory effect of JQ1 on tumor cells was clear in this setting. Our previous studies have shown that hBM-MSCs promote tumor angiogenesis through paracrine signaling, and Rahl et al. found that upregulating c-Myc also promotes tumor angiogenesis [20]. After pretreatment of tumor cells, the upregulation of c-Myc induced by hBM-MSC-CM cannot be maintained for a long time. Though the upregulation of $\mathrm{c}-\mathrm{Myc}$ in tumor tissue was 


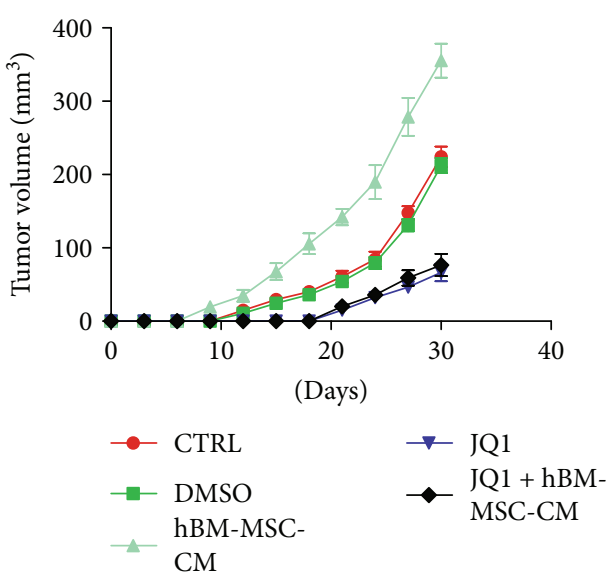

(a)

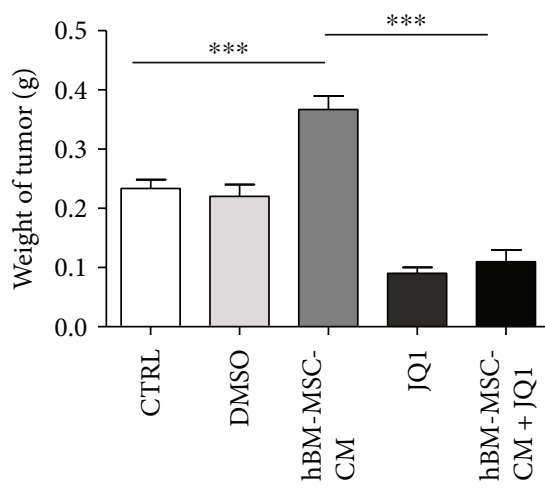

(b)

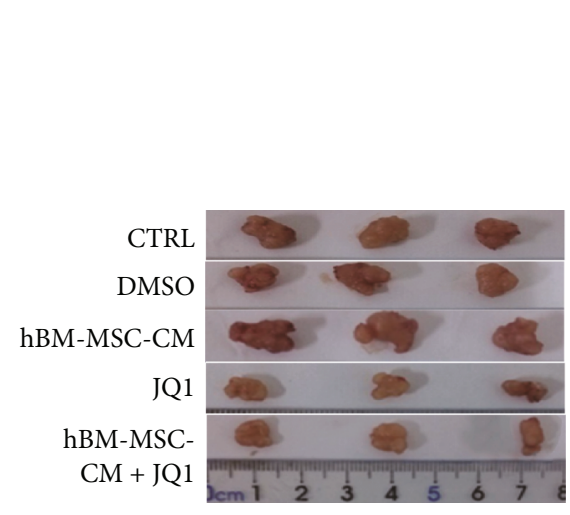

(c)
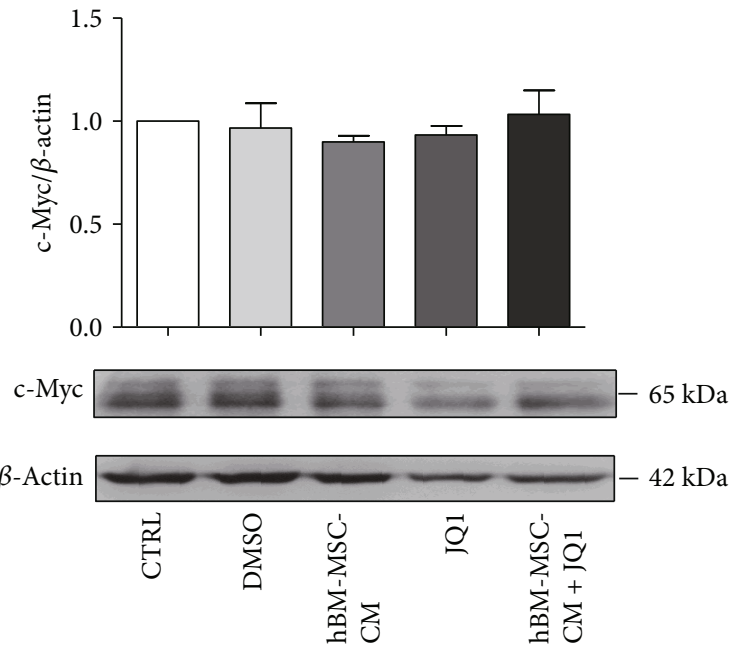

(d)

FiguRE 5: hBM-MSC-CM increased in vivo gastric tumor growth by upregulating c-Myc expression. (a) Tumor volumes of MGC-803 xenografts in immunocompromised mice. Tumor volumes of the JQ1 and hBM-MSC-CM + JQ1 groups were reduced compared with those of the hBM-MSC-CM and control groups; ${ }^{* * *} P<0.001$ by Kruskal-Wallis $H$ test. (b, c) Comparison of tumor weights in each group at day 30; ${ }^{* * *} P<0.001$ by Kruskal-Wallis $H$ test. (d) Expression of c-Myc in MGC-803 xenograft tissues of the different treatment groups was also detected with Western blot.

not maintained, the upregulation of c-Myc in tumor cells has the effect of initiating tumor development in mice. HBM-MSC-CM upregulated the expression of c-Myc in tumor cells, which may lead to reprogramming of tumor cells and affect tumor growth by promoting angiogenesis or the increase of glycolysis in vivo. This effect does not depend on the long-term expression of c-Myc. We now hypothesize that hBM-MSC-CM promotes tumor angiogenesis via upregulation of $\mathrm{c}-\mathrm{Myc}$, and thus, there are both cellintrinsic and cell-extrinsic in vivo effects of hBM-MSC-CM that increase tumor growth. We will continue to explore these processes in future studies. In conclusion, our data show that hBM-MSC-CM in the tumor microenvironment increases c-Myc expression in tumor cells, which directly promotes tumor cell proliferation and increases vascularization.

JQ1 is a small selective bromodomain and extraterminal (BET) motif inhibitor that can inhibit c-Myc expression and tumor growth $[21,22]$. Other studies have reported that a critical mechanism of JQ1 in suppressing tumor growth is its ability to block c-Myc expression [23-26]. We found that JQ1 inhibits the expression of c-Myc. Moreover, hBM-MSC$\mathrm{CM}$ promoted glucose uptake and cell migration via upregulating c-Myc in gastric cancer, both of which were suppressed by JQ1. Finally, in vivo studies showed that JQ1 inhibited the increased growth of gastric cancer xenografts that was induced by hBM-MSC-CM. Clinical trials have proven that hBM-MSCs do not undergo tumorigenesis $[4,6]$. But tumor occurrence is the result of variety of tumor-promoting factors. However, BM-MSCs are immunosuppressive, and factors that affect the immune system should be a concern with regard to tumorigenesis [27, 28]. In the present study, we found that hBM-MSC-CM can promote tumor cell proliferation, migration, and glucose uptake via upregulating $\mathrm{c}-\mathrm{Myc}$. Although there are no reports of 
hBM-MSCs causing tumor formation, their potential to promote tumorigenesis is worthy of concern for potential applications in tissue engineering.

\section{Conclusions}

hBM-MSC-CM promotes gastric cancer cell proliferation by upregulating c-Myc. c-Myc inhibitors may be effective at preventing the protumor effects of hBM-MSC-CM and, therefore, could solve the clinical safety issues of hBM-MSCs.

\section{Abbreviations}

$\begin{array}{ll}\text { MSCs: } & \text { Mesenchymal stem cells } \\ \text { hBM-MSCs: } & \text { Human bone marrow MSCs } \\ \text { hBM-MSC-CM: } & \text { hBM-MSC-conditioned medium } \\ \text { BRDs: } & \text { Bromodomain-containing proteins } \\ \text { PBS: } & \text { Phosphate-buffered saline } \\ \text { FBS: } & \text { Fetal bovine serum } \\ \text { DMEM: } & \text { Dulbecco's modified Eagle's medium } \\ \text { BET: } & \text { Bromodomain and extraterminal motif. }\end{array}$

\section{Data Availability}

The data used to support the findings of this study are included within the article.

\section{Ethical Approval}

This study and the consent procedure were approved by the local ethics committee of Jiangsu University, China. The procedure for deriving bone marrow cells from healthy donors was approved by the local ethics committee of the Affiliated Hospital of Jiangsu University, China. Informed consent was given by all donors.

\section{Conflicts of Interest}

The authors declare that they have no conflicts of interests.

\section{Authors' Contributions}

Bin Chen and Jing Yu participated in the experimental design and manuscript writing and performed the experiments. Yuanyuan Zhao, Li Sun, and Qianqian Wang were involved in establishing the animal models and manuscript. Bo Shen and Changgen $\mathrm{Xu}$ contributed to the study design and provided fresh bone marrow samples. Xiangdong Zhao participated in data analysis and cell culture. Wenrong $\mathrm{Xu}$ and Mei Wang contributed to the study design and manuscript guidance. Wei Zhu participated in the experimental design, manuscript writing, manuscript revision, and data analysis.

\section{Acknowledgments}

This work was supported by the National Natural Science Foundation of China (Grant no. 81472334), Jiangsu Province's Project of Key Research and Development Plan (Social Development) (Grant no. BE2017694), the Nanjing Science and Technology Commission (Grant no. 201605005), and the Jiangsu Provincial Health and Family Planning Commission (Grant no. LGY 2016026).

\section{References}

[1] N. Naftali-Shani, L. P. Levin-Kotler, D. Palevski et al., "Left ventricular dysfunction switches mesenchymal stromal cells toward an inflammatory phenotype and impairs their reparative properties via toll-like receptor-4," Circulation, vol. 135, no. 23, pp. 2271-2287, 2017.

[2] J. Zhan, Y. Li, J. Yu et al., "Culture medium of bone marrowderived human mesenchymal stem cells effects lymphatic endothelial cells and tumor lymph vessel formation," Oncology Letters, vol. 9, no. 3, pp. 1221-1226, 2015.

[3] W. Zhu, W. Xu, R. Jiang et al., "Mesenchymal stem cells derived from bone marrow favor tumor cell growth in vivo," Experimental and Molecular Pathology, vol. 80, no. 3, pp. 267-274, 2006.

[4] P. H. Lee, J. W. Kim, O. Y. Bang, Y. H. Ahn, I. S. Joo, and K. Huh, "Autologous mesenchymal stem cell therapy delays the progression of neurological deficits in patients with multiple system atrophy," Clinical Pharmacology and Therapeutics, vol. 83, no. 5, pp. 723-730, 2008.

[5] L. Perico, M. Morigi, C. Rota et al., "Human mesenchymal stromal cells transplanted into mice stimulate renal tubular cells and enhance mitochondrial function," Nature Communications, vol. 8, no. 1, p. 983, 2017.

[6] S. Wakitani, T. Okabe, S. Horibe et al., "Safety of autologous bone marrow-derived mesenchymal stem cell transplantation for cartilage repair in 41 patients with 45 joints followed for up to 11 years and 5 months," Journal of Tissue Engineering and Regenerative Medicine, vol. 5, no. 2, pp. 146-150, 2011.

[7] L. Wang, L. Wang, X. Cong et al., "Human umbilical cord mesenchymal stem cell therapy for patients with active rheumatoid arthritis: safety and efficacy," Stem Cells and Development, vol. 22, no. 24, pp. 3192-3202, 2013.

[8] W. Zhu, L. Huang, Y. Li et al., "Mesenchymal stem cellsecreted soluble signaling molecules potentiate tumor growth," Cell Cycle, vol. 10, no. 18, pp. 3198-3207, 2011.

[9] W. Zhu, L. Huang, Y. Li et al., "Exosomes derived from human bone marrow mesenchymal stem cells promote tumor growth in vivo," Cancer Letters, vol. 315, no. 1, pp. 28-37, 2012.

[10] A. Henssen, K. Althoff, A. Odersky et al., "Targeting MYCNdriven transcription by BET-bromodomain inhibition," Clinical Cancer Research, vol. 22, no. 10, pp. 2470-2481, 2016.

[11] J. Li, B. Fang, F. Kinose et al., "Target identification in small cell lung cancer via integrated phenotypic screening and activity-based protein profiling," Molecular Cancer Therapeutics, vol. 15, no. 2, pp. 334-342, 2016.

[12] S. Zhu, P. Sun, Y. Zhang, L. Yan, and B. Luo, "Expression of c-myc and PCNA in Epstein-Barr virus-associated gastric carcinoma," Experimental and Therapeutic Medicine, vol. 5, no. 4, pp. 1030-1034, 2013.

[13] J. Wang, Z. Liu, Z. Wang et al., “Targeting c-Myc: JQ1 as a promising option for c-Myc-amplified esophageal squamous cell carcinoma," Cancer Letters, vol. 419, pp. 64-74, 2018.

[14] Y. Jung, J. K. Kim, Y. Shiozawa et al., "Recruitment of mesenchymal stem cells into prostate tumours promotes metastasis," Nature Communications, vol. 4, no. 1, p. 1795, 2013.

[15] W. Li, Y. Zhou, J. Yang et al., "Gastric cancer-derived mesenchymal stem cells prompt gastric cancer progression through 
secretion of interleukin-8," Journal of Experimental \& Clinical Cancer Research, vol. 34, no. 1, p. 52, 2015.

[16] F. Djouad, C. Bony, F. Apparailly, P. Louis-Plence, C. Jorgensen, and D. Noël, "Earlier onset of syngeneic tumors in the presence of mesenchymal stem cells," Transplantation, vol. 82, no. 8, pp. 1060-1066, 2006.

[17] A. E. Karnoub, A. B. Dash, A. P. Vo et al., "Mesenchymal stem cells within tumour stroma promote breast cancer metastasis," Nature, vol. 449, no. 7162, pp. 557-563, 2007.

[18] C. V. Dang, "C-Myc target genes involved in cell growth, apoptosis, and metabolism," Molecular and Cellular Biology, vol. 19, no. 1, pp. 1-11, 1999.

[19] X. Fang, W. Zhou, Q. Wu et al., "Deubiquitinase USP13 maintains glioblastoma stem cells by antagonizing FBXL14mediated Myc ubiquitination," The Journal of Experimental Medicine, vol. 214, no. 1, pp. 245-267, 2017.

[20] P. B. Rahl, C. Y. Lin, A. C. Seila et al., "C-Myc regulates transcriptional pause release," Cell, vol. 141, no. 3, pp. 432-445, 2010.

[21] P. L. Garcia, A. L. Miller, K. M. Kreitzburg et al., "The BET bromodomain inhibitor JQ1 suppresses growth of pancreatic ductal adenocarcinoma in patient-derived xenograft models," Oncogene, vol. 35, no. 7, pp. 833-845, 2016.

[22] S. E. Lochrin, D. K. Price, and W. D. Figg, "BET bromodomain inhibitors-a novel epigenetic approach in castration-resistant prostate cancer," Cancer Biology \& Therapy, vol. 15, no. 12, pp. 1583-1585, 2014.

[23] P. Filippakopoulos, J. Qi, S. Picaud et al., "Selective inhibition of BET bromodomains," Nature, vol. 468, no. 7327, pp. 1067-1073, 2010.

[24] J. E. Roderick, J. Tesell, L. D. Shultz et al., "C-Myc inhibition prevents leukemia initiation in mice and impairs the growth of relapsed and induction failure pediatric T-ALL cells," Blood, vol. 123, no. 7, pp. 1040-1050, 2014.

[25] Q. Shao, A. Kannan, Z. Lin, B. C. Stack, J. Y. Suen, and L. Gao, "BET protein inhibitor JQ1 attenuates Myc-amplified MCC tumor growth in vivo," Cancer Research, vol. 74, no. 23, pp. 7090-7102, 2014.

[26] A. Wyce, G. Ganji, K. N. Smitheman et al., "BET inhibition silences expression of MYCN and BCL2 and induces cytotoxicity in neuroblastoma tumor models," PLoS One, vol. 8, no. 8, article e72967, 2013.

[27] G. R. Willis, A. Fernandez-Gonzalez, J. Anastas et al., "Mesenchymal stromal cell exosomes ameliorate experimental bronchopulmonary dysplasia and restore lung function through macrophage immunomodulation," American Journal of Respiratory and Critical Care Medicine, vol. 197, no. 1, pp. 104-116, 2018.

[28] L. Zanotti, R. Angioni, B. Calì et al., "Mouse mesenchymal stem cells inhibit high endothelial cell activation and lymphocyte homing to lymph nodes by releasing TIMP-1," Leukemia, vol. 30, no. 5, pp. 1143-1154, 2016. 


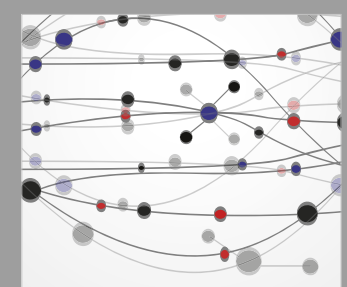

The Scientific World Journal
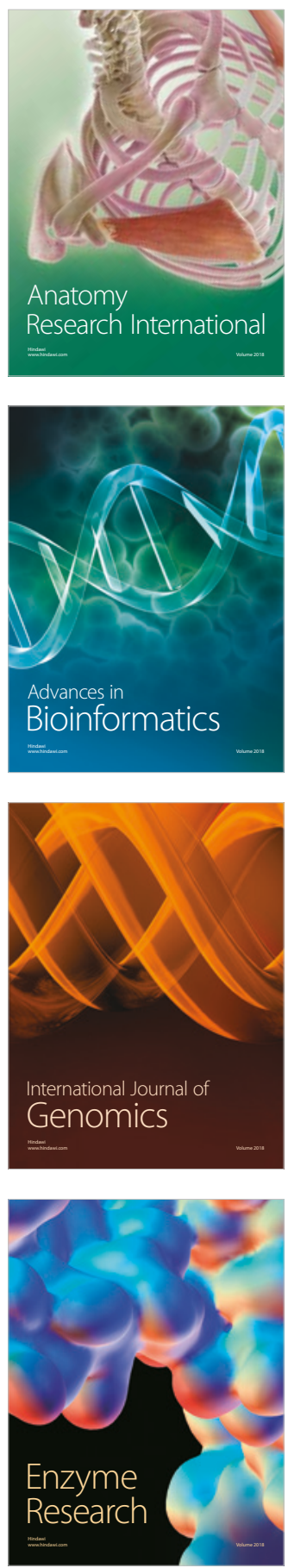
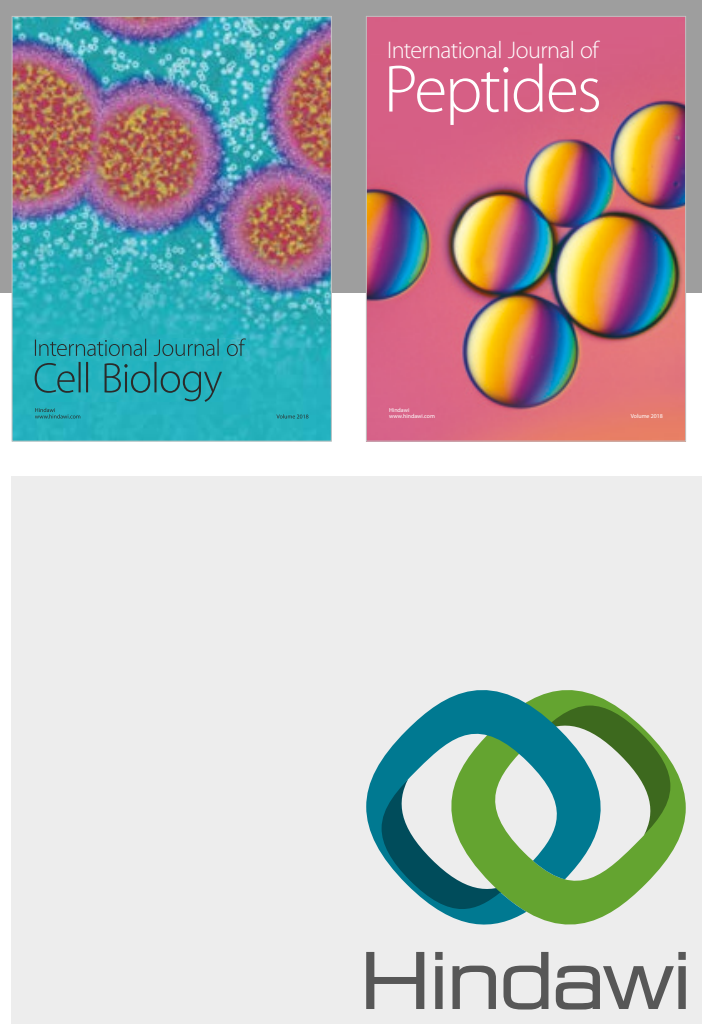

Submit your manuscripts at

www.hindawi.com
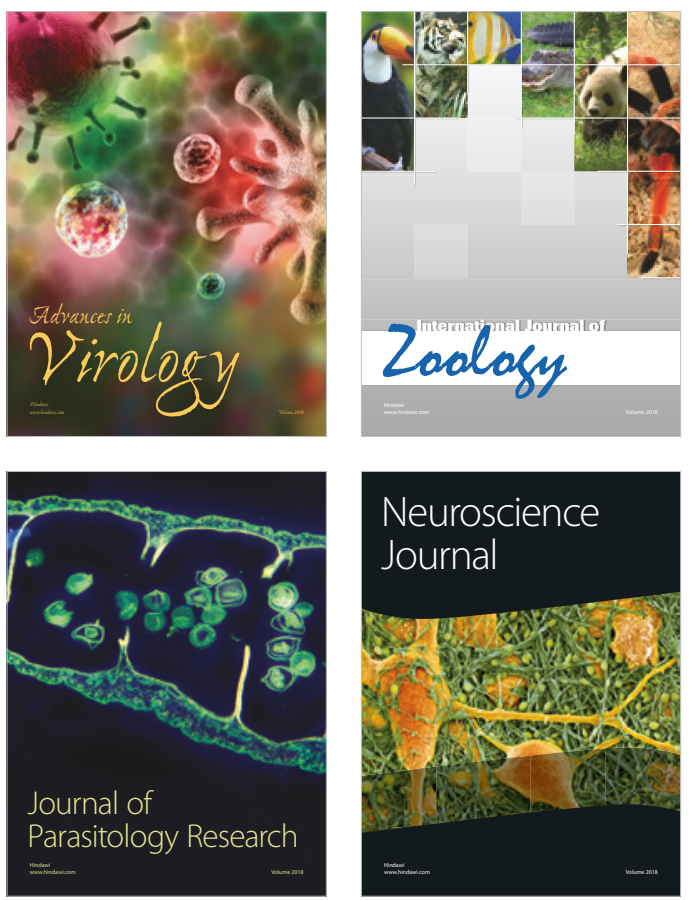
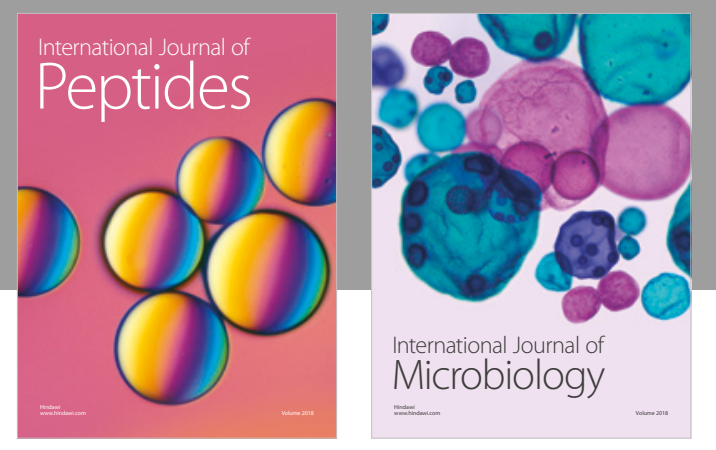

nternational Journal of Microbiology
Journal of
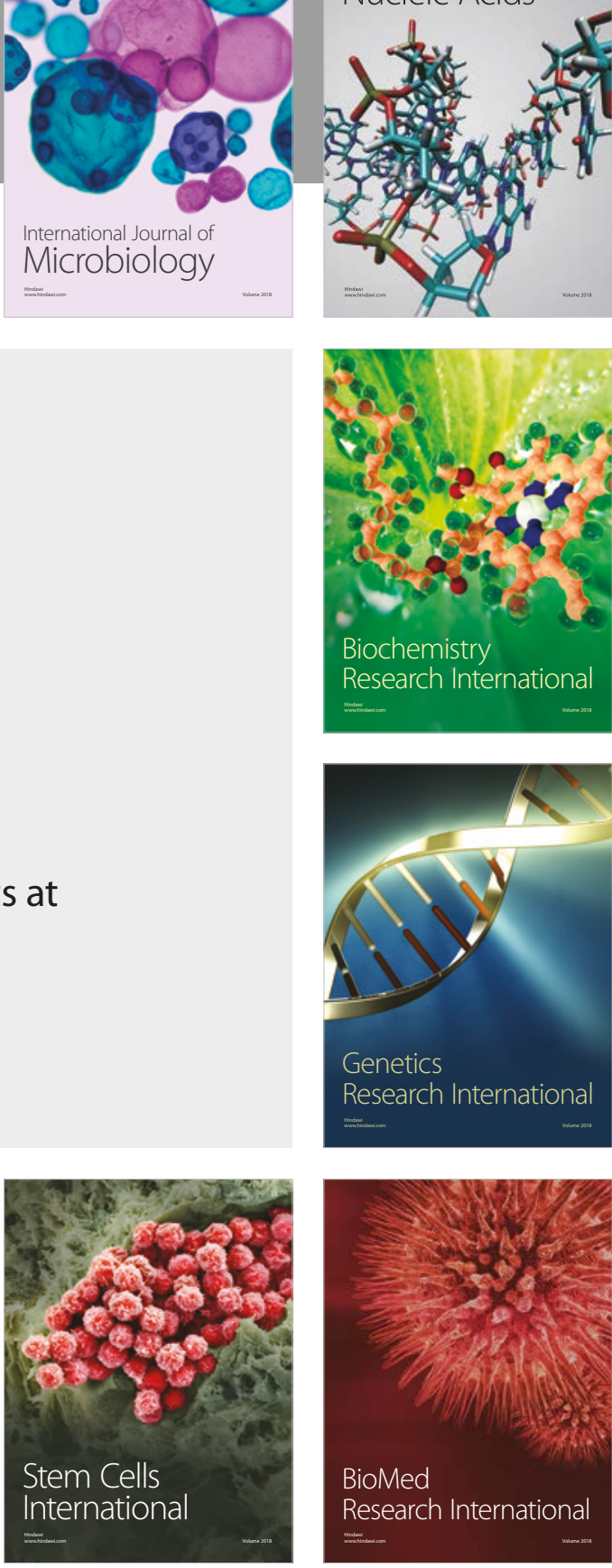
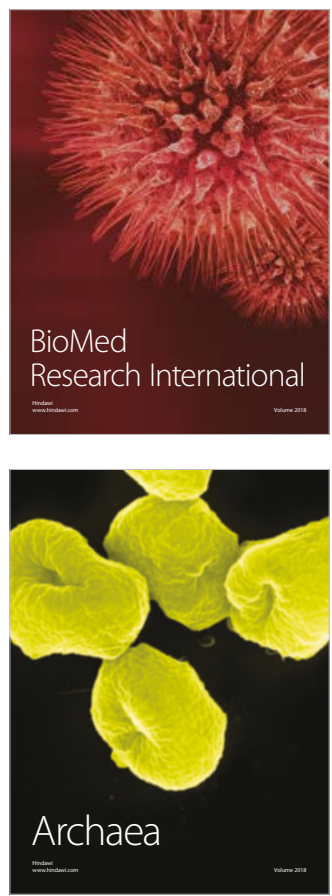